\section{NTA NEWS}

\section{Ankündigung: NTA-Jahrestreffen 2015 in Karlsruhe}

Am 26./27. November 2015 findet das Jahrestreffen des Netzwerks TA in Karlsruhe statt. Inhaltlich wird es um das Thema „Institutionelle Verankerungen von TA-Einrichtungen“" gehen. Das Jahrestreffen wird flankiert von einem erstmalig stattfindenden DirektorInnen-Treffen, von einem Workshop zum Thema „Robotik und Industrie 4.0. Wie verändert sich Arbeit?" und von einer kleinen Feier anlässlich des 20-jährigen Bestehens des ITAS.

Um was wird es inhaltlich gehen? Das „Institutionelle Setting“ einer TA-Einrichtung rückt immer wieder in den Fokus der Selbstreflexion der TA. Dies geschieht nicht nur mit Blick auf die Möglichkeit von TA, „Wirkung“ in politischen und gesellschaftlichen Debatten (TAMI-Projekt) zu erzielen, sondern auch und gerade mit Blick auf die Gründung neuer (parlamentarischer) TAEinrichtungen in Europa (PACITA). Im Rahmen der Aktivitäten des NTA wurden die unterschiedlichen institutionellen Einbettungen der TA-Einrichtungen noch nicht diskutiert, obwohl wir unter den institutionellen Mitgliedern eine Vielzahl von Varianten vorfinden: Einbettungen in die universitäre und außeruniversitäre Forschungslandschaft, in die nationalen Akademien der Wissenschaften, in die Form einer GmbH oder auch als Verein. Selbst das „TA-Machen“ als Mitwirkung in einem Forschungsprojekt, das beispielsweise der Entwicklung einer neuen Technologie dient, ist eine Form der Institutionalisierung von TA.

Ausgeführt werden diese - oft auch unter ELSI firmierenden - Arbeitspakete in Forschungsprojekten allerdings oft von Einrichtungen, die sich vermutlich nicht als TA-Einrichtung verstehen: STS-Institute, Technikphilosophie an Universitäten, Ingenieurbüros, Bürgerbeteiligungsagenturen, etc. Fragen, die damit verbunden sind: Welche Vorteile und welche Nachteile ergeben sich aus den unterschiedlichen Formen der Institutionalisierung? Gibt es optimale institutionelle Einbettungen für verschiedene Ansätze von TA, wie parlamentarische TA, constructive TA oder auch partizipative TA? Welche Distanz zum politischen Prozess, welche Distanz zum Technikentwicklungsprozess ist die Richtige? Gibt es Rechtsformen die für eine TA-Einrichtung besonders geeignet sind?

\section{Ablauf (Entwurf)}

Donnerstag, 26. November 2015

10:00 Uhr: öffentliche Sitzung der Arbeitsgruppe IuK des NTA, u. a. zum Fachportal openTA

12.30 Uhr: DirektorInnen-Treffen

14.30 Uhr: Jahrestreffen NTA 2015

16.00 Uhr: Kaffeepause

16.30 Uhr: Impulsvorträge und Diskussion: Institutionalisierte TA

19 Uhr: Sektempfang anlässlich 20 Jahre ITAS

\section{Freitag, 27. November 2015}

Workshop „Robotik, Industrie 4.0 und Arbeit“: Bereits zugesagt sind Impulsvorträge vom niederländischen Rathenau-Institut (Robot Society), vom ITA Wien (Industrie 4.0.), der TA Swiss und dem TAB (beide auf digitales Arbeiten ausgerichtet). Ein detailliertes Programm wird veröffentlicht werden unter http://www.openta.net/ aktuelles-aus-dem-nta

\section{DirektorInnen-Treffen des NTA}

Die Anregung des letzten Jahrestreffens in Wien aufnehmend, wird erstmals ein Treffen der Direktorinnen und Direktoren der institutionellen Mitglieder des NTA stattfinden. Es dient dem Austausch über den Nutzen, den das NTA für die institutionellen Mitglieder darstellt, und über die Erwartungen die seitens der TA-Einrichtungen an das Netzwerk gerichtet werden. Das Treffen findet unmittelbar vor dem Jahrestreffen 2015 statt.

\section{Kontakt zum Sprecher des NTA-Koordinationsteams}

Prof. Dr. Michael Decker

Institut für Technikfolgenabschätzung und

Systemanalyse (ITAS)

Karlsruher Institut für Technologie (KIT)

Karlstraße 11, 76133 Karlsruhe

Tel.: +49 721 608-23007

E-Mail: Michael.Decker@kit.edu 